\title{
On the robust estimation of small failure probabilities for strong non-linear models
}

\author{
Matthias Faes ${ }^{\mathrm{a}, *}$, Jonathan Sadeghic ${ }^{\mathrm{c}}$, Matteo Broggi ${ }^{\mathrm{b}}$, Marco de Angelis ${ }^{\mathrm{c}}$, \\ Edoardo Patelli ${ }^{\mathrm{c}}$, Michael Beer ${ }^{\mathrm{b}, \mathrm{c}, \mathrm{d}}$, David Moens ${ }^{\mathrm{a}}$ \\ ${ }^{a}$ KU Leuven, Department of Mechanical Engineering, Technology campus De Nayer, Jan \\ De Nayerlaan 5, St.-Katelijne-Waver, Belgium \\ ${ }^{b}$ Leibniz Universität Hannover, Institute for Risk and Reliability, Callinstrasse 34, \\ Hannover, Germany \\ ${ }^{c}$ University of Liverpool, Institute for Risk and Uncertainty, Peach Street, L69 7ZF \\ Liverpool, United Kingdom \\ ${ }^{d}$ Tongji University, International Joint Research Center for Engineering Reliability and \\ Stochastic Mechanics, Shanghai 200092, China
}

\begin{abstract}
Structural reliability methods are nowadays a cornerstone for the design of robustly performing structures, thanks to advancements in modeling and simulation tools. Monte-Carlo based simulation tools have been shown to provide the necessary accuracy and flexibility. While standard Monte-Carlo estimation of the probability of failure is not hindered in its applicability by approximations or limiting assumptions, it becomes computationally unfeasible when small failure probability needs to be estimated, especially when the underlying numerical model evaluation is time consuming.

In this case, variance reduction techniques are commonly employed, allowing for the estimation of small failure probabilities with a reduced number of samples and model calls. As a competing approach to variance reduction
\end{abstract}

\footnotetext{
*Corresponding author

Email address: matthias.faes@kuleuven.be (Matthias Faes)
} 
techniques, surrogate models can be used to substitute the computationally expensive model and performance function with an easy to evaluate numerical function calibrated through a supervised learning procedure. Both these tools provide accurate results for structural application. However, particular care should be taken into account when the reliability problems deal with high dimensional or strongly non-linear structural performances since the accuracy of the estimate is largely dependent on choices made during the surrogate modeling process. In this work, we compare the performance of the most recent state-of-the-art advance Monte-Carlo techniques and surrogate models when applied to strongly non-linear performance functions. This will provide the analysts with an insight to the issues that could arise in these challenging problems and help to decide with confidence on which tool to select in order to achieve accurate estimation of the failure probabilities within feasible times with their available computational capabilities.

Keywords: Kriging, Interval Predictor, Failure Probability, surrogate modeling, model emulation

\section{INTRODUCTION}

Nowadays, the design of engineering structures, systems or networks is largely based on computer based work flows. These work flows are particularly crafted on the application of numerical methods for the solution of the sets of differential equations that model and describe the physical processes involved in such applications. However, since these methods do not traditionally account for the inherent and unavoidable non-deterministic nature of the modeled processes, a large degree of over-conservatism needs to be 
included to prevent premature failure of the structure (i.e., the structure is no longer capable of fulfilling its initial design purpose). This conservatism might possibly cancel out the improvements achieved through the numerical optimization procedures.

Therefore, nowadays engineering design processes should account for the non-determinism in e.g., the mechanical properties of the used materials, the loading of the structure, etc. Then, based on a solid mathematical description of these properties, the reliability of these structures can be effectively assessed and included even in the earliest design stages. In practice, the assessment of the reliability is made by computing the probability that the structure is failing to satisfy its initial design requirements given the randomness or uncertainty on its structural properties and functional loading environment. Consider a model $m: \mathbb{R}^{n_{x}} \mapsto \mathbb{R}^{n_{y}}$ that predicts the structural responses $\boldsymbol{y} \in \mathcal{Y} \subset \mathbb{R}^{n_{y}}$, based on a vector of parameters $\boldsymbol{x} \in \mathcal{X} \subset \mathbb{R}^{n_{x}}$ of the model. $\mathcal{X}$ is herein the set of admissible model parameters, As the actual value of the model parameters in $\boldsymbol{x}$ is either inherently variable, unknown or both, also the prediction of the model responses $\boldsymbol{y}$ is also not deterministic. In a probabilistic context, both quantities are modeled as a random vectors, and their realizations are respectively distributed according to the probability density functions $f_{\boldsymbol{X}}(\boldsymbol{x})$ and $f_{\boldsymbol{Y}}(\boldsymbol{y})$. In that case, the probability of failure $P_{f}$, that is, the probability that the structure does not satisfy its performance requirements, is computed as the probability of a model response belonging to the failure domain $\mathcal{F}$ :

$$
P_{f}=P(\boldsymbol{y} \in \mathcal{F})=\int_{\mathbb{R}^{n y}} \mathbb{I}_{\mathcal{F}}(\boldsymbol{y}) f_{\boldsymbol{Y}}(\boldsymbol{y}) d y
$$


with $\mathbb{I}_{\mathcal{F}}: \mathbb{R}^{n_{y}} \mapsto\{0,1\}$ the indicator function, which is defined as:

$$
\mathbb{I}_{\mathcal{F}}=\left\{\begin{array}{l}
0 \\
\Longleftrightarrow \boldsymbol{Y} \in\{\boldsymbol{y} \mid \boldsymbol{y}=m(\boldsymbol{x}), \boldsymbol{x} \in \mathcal{X}, g(\boldsymbol{y})>0\} \\
1 \Longleftrightarrow \boldsymbol{Y} \in\{\boldsymbol{y} \mid \boldsymbol{y}=m(\boldsymbol{x}), \boldsymbol{x} \in \mathcal{X}, g(\boldsymbol{y}) \leq 0\}
\end{array}\right.
$$

with $g(\boldsymbol{y}): \mathbb{R}^{n_{y}} \mapsto \mathbb{R}$ the so-called limit-state function that indicates whether or not the structure satisfies a predefined performance. In practice, the considered model $m()$ can be high-dimensional in terms of parameters and responses. Moreover, the indicator function $\mathbb{I}_{\mathcal{F}}$ is in most cases non-linear. Therefore, it is generally intractable to obtain an analytical solution to the integral in eq. (1). As a solution hereto, simulation methods are commonly applied to approximate the probability of failure, based on a large number of realizations of the non-deterministic parameters $\boldsymbol{x}$ and obtaining the corresponding model responses $\boldsymbol{y}$. The most common approach is to follow Monte Carlo integration of eq. (1). However, when a sufficiently accurate estimation of a very small $P_{f}$ (i.e. $P_{f}<10^{-3}$ ) is desired (e.g. with a coefficient of variation of less than 5\%), a very high number of evaluations of the model $m()$ is typically needed, which is computationally intractable in case even medium-scaled numerical models are considered. As an attempt to alleviate this problem, advanced Monte Carlo methods, also known as variance reduction techniques, such as Line Sampling [1], Subset simulation [2], and more recently SubSet- $\infty$ [3] have been introduced. These methods have been applied to large scale problems in e.g. $[4,5,6]$, and the gain in computational efficiency has been numerously illustrated (e.g., [7]). Although these highly advanced methods typically require less model evaluations as compared to standard $\mathrm{MC}$, they still prove to be insufficiently accurate in case $\mathbb{I}_{\mathcal{F}}\left(\boldsymbol{y}_{i}\right)$ is highly non-linear. In that case, still a large number of evaluations are 
typically necessary to obtain a sufficiently small variance of the estimator.

As an alternative approach to alleviate the computational expense, the functional relation of the full model $m()$ is commonly approximated by a less computationally intensive surrogate model $\hat{\boldsymbol{y}}=\hat{m}(\boldsymbol{x})$. Such a surrogate model aims at approximating the numerical procedure of the full model $m($ ) with simple mathematical relationships, which takes less computational effort to evaluate than the solution of the model. The mathematical relationships of the surrogate model are calibrated by providing a supervised learning algorithm with $\boldsymbol{x}-\boldsymbol{y}$ pairs, obtained from a limited number of runs of $m()$, with the target of minimizing a certain norm of the prediction error (e.g., $\|\boldsymbol{y}-\hat{\boldsymbol{y}}\|_{2}^{2}$ ) of the model. The accuracy of $\hat{m}$ is commonly assessed by computing the prediction error over $\boldsymbol{x}-\boldsymbol{y}$ pairs that did not belong to the training data set. Many types of surrogate models, including Polynomial Chaos Expansions [8], Support Vector Machines [9], Neural Networks [10, 11], and many other techniques have been introduced and applied in recent years. However, since a less complicated relationship $\hat{m}$ is applied to predict $\boldsymbol{y}$, the surrogate approximation introduces a prediction uncertainty to the model response $\boldsymbol{y}[12]$. Consequently, this prediction uncertainty propagates to uncertainty concerning the computed probability of failure, that has to be effectively estimated and accounted for in such approximated analyses.

This work therefore presents a systematic approach to consider such prediction uncertainty in the estimation of small failure probabilities in nonlinear models. Specifically, Kriging and Interval Predictor Models are considered, as they readily provide an analyst with an estimate of their prediction uncertainty. A small analytic case study is performed to illustrate the proposed 
approach. This paper is an extension of [13], as it deepens the theoretical foundations of the work on both interval predictor models and adaptive Kriging and presents a more thorough case study of the approaches.

\section{Uncertain surrogate model predictions}

This section provides an overview of the considered surrogate modeling techniques that are considered in this paper: Kriging and Interval Predictor models. Since these models provide the analyst with an estimate of the uncertainty on the prediction of the model response, such uncertainty in the model output will propagate to the computed probabilities of failure in the form of bounds of the estimation.

\subsection{Kriging}

Kriging, also commonly referred to as Gaussian Process Modeling, approximates the full model $m()$ as the sum of a functional regression model $F(\beta, \boldsymbol{x})$, where $F$ is usually a polynomial function and $\beta$ indicating the re-

gression terms, and a stationary zero-mean Gaussian stochastic process $z(\boldsymbol{x})$ [14]. Formally, the Kriging surrogate model $\hat{m}_{K r}()$ for the $l^{\text {th }}$ response is expressed as:

$$
\hat{y}_{l}=\hat{m}_{l, K r}(\boldsymbol{x})=F\left(\beta_{:, l}, \boldsymbol{x}\right)+z_{l}(\boldsymbol{x})
$$

with $l=1, \ldots, n_{y}$. As such, a single Kriging model is constructed for each separate response. For the remainder of the paper, index $l$ is omitted for the sake of notational simplicity. When a vector of model responses is considered, it is implicitly implied that a single Kriging model was constructed for each 
response. In eq. (3), the polynomial regression model is given as the linear superposition of a number of functions $f(\boldsymbol{x}): \mathbb{R}^{n} \mapsto \mathbb{R}$ :

$$
F(\beta, \boldsymbol{x})=\boldsymbol{f}^{T}(\boldsymbol{x}) \boldsymbol{\beta}
$$

where $\boldsymbol{\beta}$ are the corresponding regression coefficients that have to be estimated. The auto-covariance of the stationary zero-mean Gaussian stochastic process $z(\boldsymbol{x})$ is given as:

$$
E\left[z\left(\boldsymbol{x}_{i}\right), z\left(\boldsymbol{x}_{j}\right)\right]=\sigma^{2} R\left(\theta, x_{i}, x_{j}\right)
$$

with $\sigma$ the process variance and $R\left(\theta, x_{i}, x_{j}\right)$ the correlation model between two $x_{i}, x_{j}$ in $\mathcal{X}$. The correlation model is characterized by a set of coefficients $\theta$.

As such, first the degree of the polynomial regression model and the correlation function family are selected by the analyst, based on expert opinion. Then, the correlation coefficients, process variance and correlation parameter $\theta$ are determined using a supervised learning procedure. Specifically, $n_{t}$ couples of model parameters $\boldsymbol{x}_{t r}$ and corresponding responses $\boldsymbol{y}_{t r}$ of the full model $m()$ are provided. Based on these couples, the necessary parameters are determined following a maximum likelihood approach $[15,16]$.

Since Kriging associates a Gaussian random variable to each predicted $\hat{\boldsymbol{y}}=\hat{m}_{K r}(\boldsymbol{x})$, also an estimation of the variance $\zeta(\boldsymbol{x})$ to the prediction is given by the Kriging model. Moreover, it can be shown that Kriging is an unbiased predictor, as it is exact (i.e., zero variance and deviation from mean) for the provided training points $\boldsymbol{x}_{t r}$. However, the variance of the prediction (and as such the uncertainty) increases when the distance $\left\|\boldsymbol{x}_{t r}-\boldsymbol{x}\right\|_{2}$ from the training points becomes larger. As such, when considering the $k \cdot \sigma$-bounds, 
with $k \in \mathbb{Z}^{+}$, the response of the Kriging predictor can as such be interpreted as an interval:

$$
\hat{\boldsymbol{y}}^{I}=\left[\hat{m}_{K r}(\boldsymbol{x})-k \cdot \zeta(\boldsymbol{x}) ; \hat{m}_{K r}(\boldsymbol{x})+k \cdot \zeta(\boldsymbol{x})\right]
$$

This interval is by definition symmetric around the deterministic estimate of the Kriging model. By applying this method for each model response $y_{l}, l=1, \ldots, n_{y}$, an interval vector $\hat{\boldsymbol{y}}^{I}$ containing the $k \cdot \sigma$ confidence intervals of the model response is obtained next to the deterministic estimate $\hat{y}_{l}$ of the model response. Note that the assumption that the discrepancy between the actual model and the regression model as a stationary zero-mean Gaussian stochastic process can only be fulfilled when the order of the chosen regression model is sufficiently similar to $m()$. In practice however, this condition is not so trivial to obtain, since $m()$ is in general unknown for the entire sample space, especially when $m()$ requires considerable computational expense to be evaluated.

A technique for adaptively refining Kriging models in the context of propagating interval uncertainty was introduced in [17]. As can intuitively be understood, there exists some similarity in accurately predicting small failure probabilities and propagating interval uncertainty: both processes need a surrogate model that is accurate in the extremes of the numerical model. The adaptive Kriging refinement presented in [17] is based on the idea of Maximum Improvement (MI) to direct the sampling of additional parts. The MI value of a certain candidate point is specifically evaluated as:

$$
\begin{aligned}
\mathrm{MI} & =\frac{\min (\tilde{m}(\boldsymbol{x}))-\left(\tilde{m}\left(\boldsymbol{x}_{\text {new }}\right)-\Delta \tilde{m}\left(\boldsymbol{x}_{\text {new }}\right)\right)}{\min (\tilde{m}(\boldsymbol{x}))} \\
\mathrm{MI} & =\frac{\left(\tilde{m}\left(\boldsymbol{x}_{\text {new }}\right)+\Delta \tilde{m}\left(\boldsymbol{x}_{\text {new }}\right)\right)-\max (\tilde{m}(\boldsymbol{x}))}{\max (\tilde{m}(\boldsymbol{x}))}
\end{aligned}
$$


with $\boldsymbol{x}_{\text {new }}$ the candidate sample point and $\Delta m_{\text {new }}$ the Kriging estimate of the variance at this point (i.e., the $k \cdot \sigma$ bound). Starting from a coarse large space-filling design, an initial Kriging model is trained. Then, based on the MI metric, sampled over a very fine space-filling design, a set of new points is selected and appended to the initial design before retraining the new Kriging model. This procedure is repeated until convergence of MI [17].

\subsection{Interval Predictor Model}

Conversely to most surrogate modeling approaches, Interval Predictor Models (IPM) provide the analyst with a set-valued mapping $m_{I P M}^{I}: \boldsymbol{x} \mapsto$ $\boldsymbol{y}^{I} \subset \mathcal{Y}$, instead of only one crisp value [18, 19]. Specifically, the IPM translates the crisp valued vector of input parameters $\boldsymbol{x}$ to an interval vector $\boldsymbol{y}^{I}$ bounding the range of the actual crisp model prediction. This interval vector $\boldsymbol{y}^{I}$ is defined as:

$$
\hat{\boldsymbol{y}}^{I}=\left\{\boldsymbol{y} \mid \boldsymbol{y}=p^{T} \cdot \phi(\boldsymbol{x}), \boldsymbol{p} \in \boldsymbol{p}^{I}\right\}
$$

with $\boldsymbol{\phi}(\boldsymbol{x})$ a suitable polynomial basis with predefined order $d, p \in \mathbb{R}^{d}$ a vector containing the expansion parameters for the polynomial basis and apex $T$ denoting the vector transpose operation. The parameters $\boldsymbol{p}$ are determined by providing $n_{t}$ couples of model parameters $\boldsymbol{x}$ and corresponding responses $\boldsymbol{y}$ of the full model $m()$. The set $\boldsymbol{p}$ can be chosen to be hyper-rectangular which enables the numerical training scheme to be simplified [20]. Then, instead of determining a single set of crisp parameters $\boldsymbol{p}$, the training of the IPM consists of determining the boundaries (i.e. $\underline{p}$ and $\bar{p}$ ) such that all $(\boldsymbol{x}, \boldsymbol{y})$ are encapsulated by the predicted intervals of the IPM. Fortunately, this means that the IPM can be trained by solving an optimization program 
which is both linear and convex. This is obtained according to a constrained optimization approach where the expectancy of the interval range is minimized, while ensuring that $\underline{y}_{i}<y_{i}<\bar{y}_{i}, i=1, \ldots, n_{y}$, with $y_{i}$ in this case a training sample. If the IPM is being created for the purposes of reliability analysis it may be more useful to minimize the difference between the failure probability calculated by the upper and lower bounds $\left(\bar{P}_{f}-\underline{P}_{f}\right)$, as this will result in tighter bounds - at the consequence of having to solve a non-convex program. In addition, it is important to evaluate the objective function either analytically or with high accuracy, rather than empirically, when small samples are used to train an IPM intended for use with small failure probabilities. This is because the standard deviation of the empirical estimate of the failure probability may well be larger than the failure probability in these cases. Based on the trained IPM, the lower and upper bound, being $\underline{\boldsymbol{y}}$ and $\overline{\boldsymbol{y}}$ of the prediction interval vector $\hat{\boldsymbol{y}}^{I}$ are estimated as:

$$
\begin{aligned}
& \underline{\boldsymbol{y}}=0.5 *(\bar{p}+\underline{p})^{t} \cdot \phi(\boldsymbol{x})-0.5 *(\bar{p}-\underline{p})^{t} \cdot \phi(|\boldsymbol{x}|) \\
& \overline{\boldsymbol{y}}=0.5 *(\bar{p}+\underline{p})^{t} \cdot \phi(\boldsymbol{x})+0.5 *(\bar{p}-\underline{p})^{t} \cdot \phi(|\boldsymbol{x}|)
\end{aligned}
$$

It is clear that obtaining more data will expand the set $\boldsymbol{p}$, and without observing an infinite amount of data the obtained bounds on the model output will never be completely robust. Fortunately Scenario Optimization theory provides a framework for judging how well the model will generalize when trained with a finite set of observed data. The reliability $R$ of an IPM, i.e. the probability that a future unobserved data point is contained within the IPM, is bounded by

$$
\operatorname{Prob}_{P^{n}}[R \geq 1-\epsilon]>1-\beta
$$


where $\epsilon$ and $\beta$ are the confidence and reliability parameters, which for our hyper-rectangular model can be obtained from

$$
\beta \geq\left(\begin{array}{c}
k+d-1 \\
k
\end{array}\right) \sum_{i=0}^{k+d-1}\left(\begin{array}{c}
N \\
i
\end{array}\right) \epsilon^{i}(1-\epsilon)^{N-i},
$$

where $N$ is the number of training data points, $k$ is the number of data points discarded by some algorithm and $d$ is the dimensionality of the parameter vectors. The robustness of an IPM can be evaluated by plotting $1-\epsilon$ against $1-\beta$, which we will refer to as a confidence-reliability plot, and then finding $1-\epsilon$ for an arbitrarily high value of $1-\beta$. In simple terms, if the area under the confidence-reliability plot is larger then the IPM is more robust. Reassuringly, reducing the degrees of freedom in the meta-model $(d)$ increases this area, and hence improves the generalization of the meta-model (refer to [20] for a more thorough discussion).

The bound given in eq. (12) is overly conservative in many cases as it assumes the convex optimization program used to create the IPM is fully supported (that is, the number of support constraints, which when removed result in a tighter IPM, is equal to the number of optimization variables). In some cases this may be overly conservative, and therefore a more optimistic bound can be obtained by identifying the number of support constraints, $s$, and then applying

$$
\epsilon(s)=1-\sqrt[N-s]{\frac{\beta}{N\left(\begin{array}{l}
N \\
s
\end{array}\right)}},
$$

which is valid for non-convex programs.

Note that the IPM does not provide a crisp value of the model response. For comparison with the crisp value that is provided by the full model $m()$ 
and the Kriging predictor, the least squares estimate using the basis chosen for the IPM is used. This should be roughly similar to finding the mean of a staircase predictor model, as in [21].

In order to reduce the number of support constraints in the IPM and hence improve its reliability two strategies were adopted. Firstly we set $\bar{p}_{i}=\underline{p}_{i}$ for $i>1$, in other words the parameter vector was the same for the upper and lower bound except for a constant, which almost halves the bound on the number of support constraints. This strategy works particularly well when modeling deterministic functions. Secondly, an iterative scheme is used to refine the basis chosen. Firstly, a polynomial basis with the maximum required degree is created and then the IPM is trained. The monomial term with the lowest $p_{i}$ is removed. The IPM is now retrained with the new basis and the procedure is repeated until the IPM has a sufficiently small uncertainty.

An analogy between IPM's and interval fields [22, 23, 24] can be established in analogy to the analogy between Gaussian Random Fields and Kriging. An interval field is modeled by means of a truncated series expansion of interval scalars $\boldsymbol{p}^{\boldsymbol{I}}$ that are used to scale a set of basis functions $\phi(\mathbf{r})$ that are defined over the model domain, where the former represent the magnitude of the spatial uncertainty in the model and the latter represent the spatial nature of this uncertainty. Similar considerations can be made concerning the IPM, where instead of efficiently trying to represent the model domain, an accurate representation of the solution manifold of the numerical model is constructed. The interval valued parameters $p$ represent the uncertainty in the prediction of the model, whereas the basis functions represent the global 
shape of the solution manifold.

\subsection{Interval failure probability}

From the previous, it can be understood that both Kriging and IPM surrogate models either give an estimate of the uncertainty on the prediction of $\hat{\boldsymbol{y}}$ or provide the analyst with a set-valued response that prescribes this uncertainty. In both cases, the predicted response $\hat{\boldsymbol{y}}$ is modeled as belonging to an interval $\hat{\boldsymbol{y}}^{I}$. As such, in the context of estimation the reliability of the considered structure given a vector of random model parameters $\boldsymbol{x} \sim$ $f_{\boldsymbol{X}}(\boldsymbol{x})$, the resulting random model responses can be regarded as belonging to a probability box $[\hat{\boldsymbol{y}}]$ due to the superposition of the interval uncertainty from the surrogate model on the probabilistic description of the response $\boldsymbol{y}$ stemming from the random model parameters $\boldsymbol{x}$. As such, in the context of determining the structural reliability, also the probability of failure $\hat{P}_{f}$ becomes interval valued. Specifically, $\hat{P}_{f}^{I}$ can be computed as:

$$
\hat{P}_{f}^{I}=\int_{\mathbb{R}^{n_{y}}} \mathbb{I}_{\mathcal{F}}([\hat{\boldsymbol{y}}]) f_{\hat{\boldsymbol{Y}}^{\boldsymbol{I}}}^{I}([\hat{\boldsymbol{y}}]) d[\hat{\boldsymbol{y}}]
$$

which can be solved following e.g. a nested optimization approach [25].

However in this specific context, some considerations allow for simplification of this equation. In case of Kriging, the superimposed interval uncertainty on the predicted model response is strict in the sense that the upper and lower bounds do not cross. This is a direct result from the truncation of the random variable that is associated to each predicted response. Also, since during the training of the IPM, the explicit constraint $\underline{y}_{i}<y_{i}<\bar{y}_{i}, i=1, \ldots, n_{y}$ is included, a similar observation can be made 
in this context, as demonstrated in [26] and [21]. Therefore, only the extreme bounds of the predicted response intervals need to be considered in the evaluation of the failure probability. As such, eq. (14) can be split up as [27]:

$$
\begin{aligned}
& \underline{\hat{P}}_{f}=\int_{\mathbb{R}^{n_{y}}} \mathbb{I}_{\mathcal{F}}(\underline{\hat{\boldsymbol{y}}}) \underline{f} \underline{\hat{\boldsymbol{Y}}}(\underline{\hat{\boldsymbol{y}}}) d \underline{\hat{\boldsymbol{y}}} \quad \approx \frac{1}{N_{P_{f}}} \sum_{i=1}^{N_{P_{f}}} \mathbb{I}_{\mathcal{F}}\left(\underline{\hat{\boldsymbol{y}}}_{i}\right) \\
& \hat{\bar{P}}_{f}=\int_{\mathbb{R}^{n_{y}}} \mathbb{I}_{\mathcal{F}}(\hat{\overline{\boldsymbol{y}}}) \bar{f}_{\hat{\overline{\boldsymbol{Y}}}}(\hat{\overline{\boldsymbol{y}}}) d \hat{\overline{\boldsymbol{y}}} \approx \frac{1}{N_{P_{f}}} \sum_{i=1}^{N_{P_{f}}} \mathbb{I}_{\mathcal{F}}\left(\hat{\overline{\boldsymbol{y}}}_{i}\right)
\end{aligned}
$$

where, $\underline{f}_{\underline{\boldsymbol{Y}}}(\underline{\hat{\boldsymbol{y}}})$ and $\bar{f} \hat{\overline{\boldsymbol{Y}}}(\hat{\overline{\boldsymbol{y}}})$ are respectively the distribution function of the lower and upper bounds on the prediction of the surrogate model. It should be noted that this computation only requires a single call to the surrogate model $\hat{m}()$, as both Kriging and IPM provide the analyst with the confidence bounds on the model prediction.

In case dependent random model parameters are considered, the computation of the failure probability is usually performed in standard normal space (SNS). Due to the interval-valued uncertainty that is attributed to each realization of the random model responses, also the limit state function becomes interval valued after transformation to SNS. However, it can be shown that due to the monotonicity of the iso-probabilistic transformation to SNS (see [28]), the minimum and maximum value of the limit state function correspond to the vertices of the interval-valued uncertainty on the model response realizations. Therefore, the above argumentation also holds in this case.

This method as such allows an analyst to make a robust prediction of the probability of failure of a highly non-linear, computationally demanding 
computer model at greatly reduced cost. It furthermore allows the analyst to uniquely separate the uncertainty stemming from the modeled physics from the uncertainty that stems from applying a surrogate model instead of the full numerical simulation code.

\section{Uncertain failure probability estimation}

In the study of the uncertainty concerning the estimation failure probability due to the application of surrogate modeling techniques, Adjiman's function is applied:

$$
y=f_{\text {adj }}\left(x_{1}, x_{2}\right)=\cos \left(x_{1}\right) \cdot \sin \left(x_{2}\right)-\frac{x_{1}}{\left(x_{2}^{2}+1\right)}
$$

Based on this function, decreasing levels of failure probability are estimated by considering the threshold value for $y_{t h} \in\{2,2.5,3,3.1,3.2,3.3$, 3.4, 3.5, 3.6, 3.7, 3.8, 3.9, 3.95, 4\}. In a first attempt, advanced Monte Carlo methods such as Line Sampling and SubSet simulation, as well as regular Monte Carlo simulation are applied, and their performance in terms of necessary number of function evaluations and variance of the predictor are compared. Then, different surrogate models for Adjiman's function are constructed using three techniques:

- an Interval Predictor Model, based on a $7^{\text {th }}$-order polynomial basis, refined using a basis refinement algorithm until only 12 monomials are present,

- a Kriging model with $2^{\text {nd }}$-order regression model $F(\beta, \boldsymbol{x})$ and an exponential correlation model $R\left(\boldsymbol{\theta} ; \boldsymbol{x}_{i}, \boldsymbol{x}_{j}\right)=\exp \left(-\theta\left|\boldsymbol{x}_{i}-\boldsymbol{x}_{j}\right|\right)$, 
- a Kriging model with $2^{\text {nd }}$-order regression model $F(\beta, \boldsymbol{x})$ and an exponential correlation model $R\left(\boldsymbol{\theta} ; \boldsymbol{x}_{i}, \boldsymbol{x}_{j}\right)=\exp \left(-\theta\left|\boldsymbol{x}_{i}-\boldsymbol{x}_{j}\right|\right)$, but trained using the adaptive refinement scheme from [17], as also explained in section 2.1, using an initial training set size of 10 samples and an increase of 5 additional training points per iteration,

and these surrogate models are applied to perform a large scale Monte Carlo integration of eq. (1). Both modeling techniques are applied to the same training data sets containing either 100, 250, 500 or 1000 deterministic training samples. It should be noted that no computational gain is expected in the application of a surrogate model for the considered test function. Nonetheless, it allows for conceptually comparing the accuracy in predicting small failure probabilities of the considered surrogate modeling techniques in a rigorous way.

Since the considered surrogate modeling approaches are conceptually very different, comparison of their accuracy based on some a priori (i.e., before computing $P_{f}$ ) metric is non-informative. The most obvious way would be to compute for instanc the $R^{2}$-value and the Chebyshev norm $\left(D_{c h}\right)$ of the difference between the analytical model and surrogate prediction using a set of validation data. However, since the interval predictor model only provides a set valued response for each combination of parameter values, such metrics computed over for instance the midpoint of the predicted intervals are non-informative. Hence, such comparison does not tell much about the performance of the methods. All numerical computations, except for the adaptive Kriging refinement, are performed using [29]. 


\subsection{Advanced Monte Carlo sampling}

As a first step in the analysis, the performance of Monte Carlo, Line Sampling with an adaptive algorithm to find the important direction (see [30]) and SubSet- $\infty$ [3] is tested in terms of the estimation of the failure probability, the coefficient of variance of this estimation and the number of samples that were needed to obtain the estimate. These simulation methods are applied directly using the analytical function, as introduced in eq. (16), to ensure that this analysis is not biased due to prediction errors of the surrogate models. Both $x_{1}$ and $x_{2}$ are assumed to be marginally uniform distributed within the interval $[-4 ; 4]$ with zero covariance.

The Monte Carlo and Line Sampling methods were applied until a coefficient of variance $(\mathrm{CoV})$ of the estimator of $5 \%$ was reached, albeit with a maximum of $10^{7}$ samples. Hereto, the sampling was performed in batches of $5 \cdot 10^{2}$ samples for Monte Carlo simulation and 200 lines for Line Sampling. Then, after each batch the $\mathrm{CoV}$ is estimated and the simulation is stopped if $\mathrm{CoV}<0.05$. The important direction for Line Sampling is found by means of the adaptive algorithm presented in [30]. For SubSet- $\infty$, the intermediate levels of $P_{f}$ were set to 0.1 and the initial population size was heuristically set until a sufficiently small $\mathrm{CoV}$ was obtained. A CoV of approx. $8 \%$ for the prediction of $P_{f}$ for $y_{t h}=2$ was obtained at $10^{3}$ samples, as the $\mathrm{CoV}$ did not improve significantly when the population size was further increased. The same initial population size was kept constant for all other evaluations of the failure probability.

Figure 1 illustrates the topology of the limit state function of Adjiman's function in the standard normal space $\mathcal{U}$. Herein, $u_{1}$ and $u_{2}$ respectively cor- 
respond to $u_{1}=T_{u}\left(x_{1}\right)$ and $u_{2}=T_{u}\left(x_{2}\right)$, with $T_{u}: \mathcal{X} \mapsto \mathcal{U}$ a transformation operator mapping responses from physical to standard normal space. This plot is generated by performing $5 \cdot 10^{4}$ Monte-Carlo evaluations of the analytical function, with a threshold value of $y_{t h}=3.7$. The red dots in this figure indicate the samples laying in the failure domain $\mathcal{F}$ (i.e., $\mathcal{I} \leq 0)$, whereas the samples in the safe domain $\mathcal{S}$ (i.e., $\mathcal{I}>0$ ) are indicated in green. As it may be noted, a highly non-linear notched limit state function $g(\boldsymbol{u})$ is obtained, which poses a challenge for the applied advanced Monte Carlo methods.

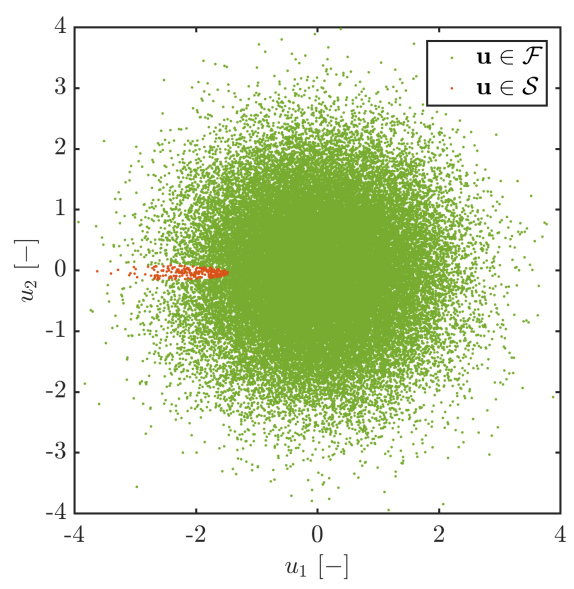

Figure 1: Failure domain $\mathcal{F}$ and safe domain $\mathcal{S}$ in standard normal space for Adjiman's function

Figure 2 shows the estimated failure probability, as obtained using Monte Carlo, Advanced Line Sampling and SubSet- $\infty$, as a function of the threshold value. First, it can be seen that the estimate of the failure probability as a function of the threshold of $y$ is approximately equal for Monte Carlo and the SubSet methods, as long as the failure probability remains moderately large (i.e., $P_{f}>10^{-3}$ ). However, the obtained results diverge significantly when smaller failure probabilities are computed. Advanced Line Sampling 
on the other hand provides in this case a better estimate for the smaller failure probabilities, which is explained by the independence of Line Sampling performance to the magnitude of the probability of failure [31].

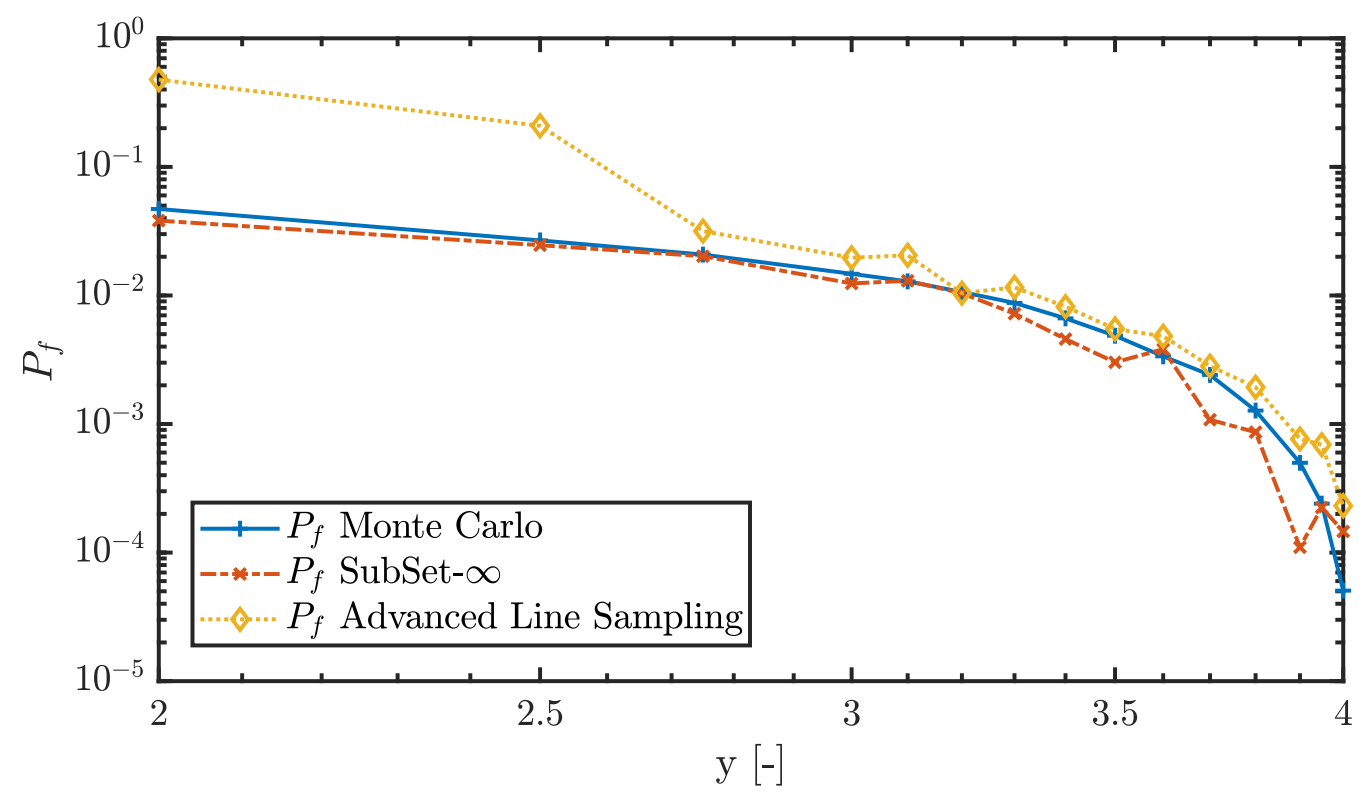

Figure 2: Estimated failure probability and the coefficient of variance for different threshold values $y$ for Adjiman's function

Figure 3 shows the $\mathrm{CoV}$ of the failure probabilities estimated by the three methods. It can be noted that the variance on the failure probability predictor that is obtained by Monte Carlo and Advanced Line Sampling is up to a factor 5 smaller as compared to SubSet- $\infty$. This is a direct result from the fact that in the case of Monte Carlo and Advanced Line Sampling, additional samples were generated until a specified $\mathrm{CoV}$ of $5 \%$ was reached, whereas the SubSet method was heuristically tuned to minimize the CoV of the prediction. Moreover, in the case of SubSet, the $\mathrm{CoV}$ measures up to $60 \%$ in the case of the smallest considered failure probabilities. 


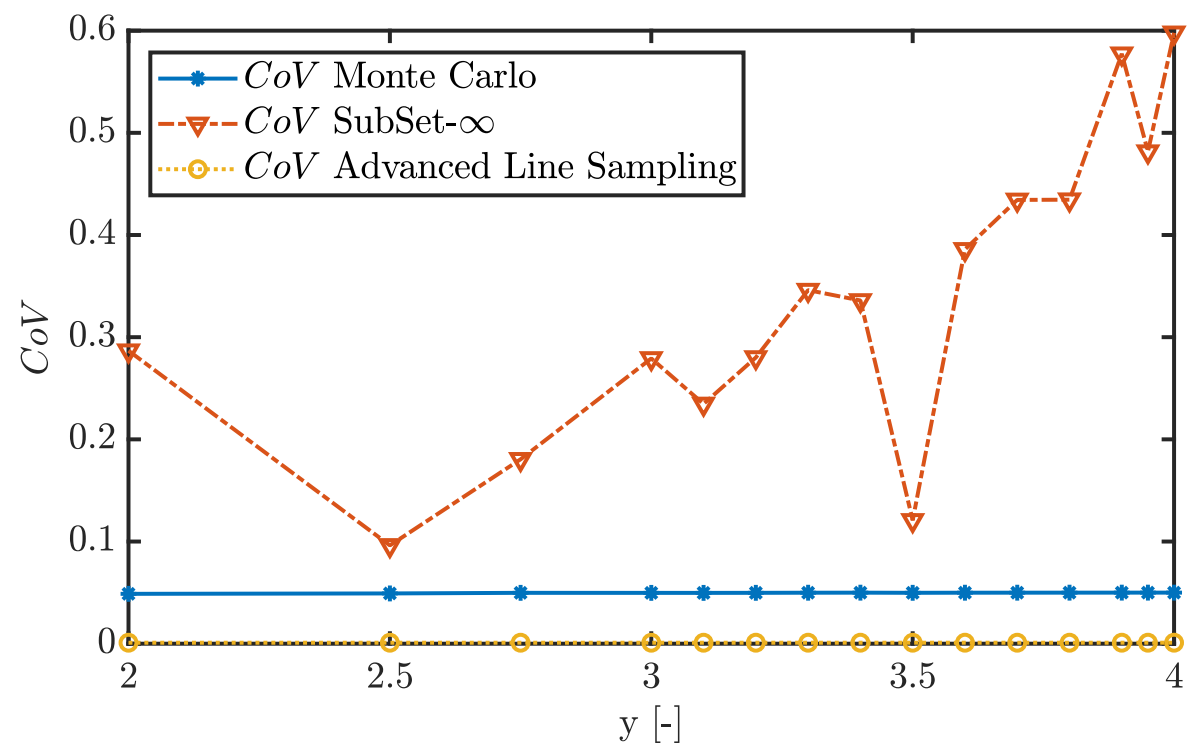

Figure 3: Estimated failure probability and the coefficient of variance for different threshold values $y$ for Adjiman's function

Figure 4 shows the computational efficiency in terms of necessary number of samples to perform the probability of failure estimate. From this figure, it is clear that SubSet- $\infty$ is more efficient than Advanced Line Sampling, which in its turn is more efficient than standard Monte Carlo simulation for the estimation of the failure probability. This is particularly true when small failure probabilities are considered. However, in that context it should be noted that the variance of the Monte Carlo estimator is an order of magnitude lower as compared to the variance of $\hat{P}_{f}$, as obtained by SubSet, which limits the credibility of the estimate. The variance of $P_{f}$ obtained via Advanced Line Sampling is approximately equal to that of Monte Carlo, albeit at a strongly reduced computational cost.

It should be noted that SubSet- $\infty$, the most efficient technique, still re- 
quires more than 2000 model evaluations, which is prohibitive when the estimation of the failure probability of a structure using computationally expensive computer models $m()$ is considered.

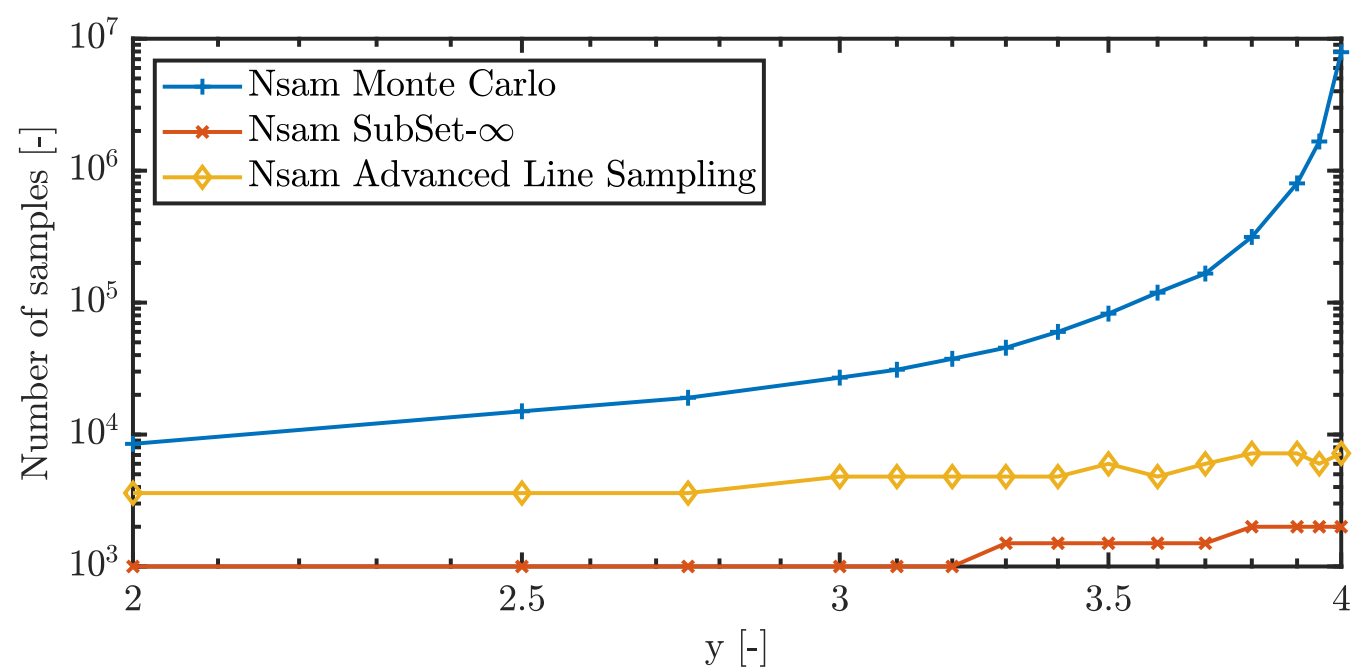

Figure 4: Number of necessary samples of the advanced Monte Carlo methods for different threshold values $y$ for Adjiman's function

As such, it can be concluded that although highly performing advanced Monte Carlo methods exist to date, the estimation of small failure probabilities in highly non-linear models still can prove to be computationally very demanding. Therefore, even using these advanced Monte Carlo methods, the application of surrogate modeling techniques still proves to be of importance, as the training of such surrogate model typically requires less model evaluations as compared to a direct application of the advanced Monte Carlo methods for the estimation of a small probability of failure. As discussed in section 2 , this however imposes uncertainty on the prediction of the failure probability as well. 


\subsection{Surrogate model based estimation}

This section presents results of the effect of the selection of the surrogate modeling approach and corresponding training on the uncertainty that is attributed to the prediction. Using the constructed surrogate models, decreasing levels of failure probability are estimated by performing Monte Carlo sampling until the $\mathrm{CoV}$ of the predictor was less than $5 \%$, analogously to the method that was applied in section 3.1.

The results for each estimation of the failure probability, for each of the constructed surrogate models is illustrated in figures $5-7$. For the Kriging models, the $2 \cdot \sigma$ bounds are considered, which yield a $95.5 \%$ confidence interval for $P_{f}$. For the IPM the uncertainty in the bounds on $P_{f}$ is considered as being less than $\epsilon$ when $\beta=1-96$. In other words the bounds on $P_{f}$

obtained from integrating over the bounds of the IPM must be expanded by $\epsilon$.

Figure 5 illustrates the performance of the regular Kriging surrogate modeling approach. Specifically, the $\pm 2 \cdot \sigma$ bounds are illustrated together with the crisp (mean) estimate of the model for all considered training data sets. Also the prediction of the failure probability using the analytic model is illustrated. First, in case sufficient data are used for the training, the regular Kriging is capable of providing a relatively accurate crisp estimate of the failure probability, as long as $P_{f}>5 \cdot 10^{-03}$. For smaller failure probabilities, Kriging fails in all cases. Second, it can be noted that the Kriging prediction is conservative in the sense that the $\pm 2 \cdot \sigma$ alway encompass the true failure probability. However, the lower bound prediction fails in all cases when $y>3.7$. This is due to the difficulty of sampling small failure probabilities 
with standard Monte Carlo with a limited sample set. Finally, when more data are included in the training of the Kriging model, the $\pm 2 \cdot \sigma$ bounds on the prediction become tighter. This is a direct result of the conditioned random field that underlies these predictions. When more points are located throughout the model domain, the relative distance between training points decreases, and as such also the variance of the predicted random variable.

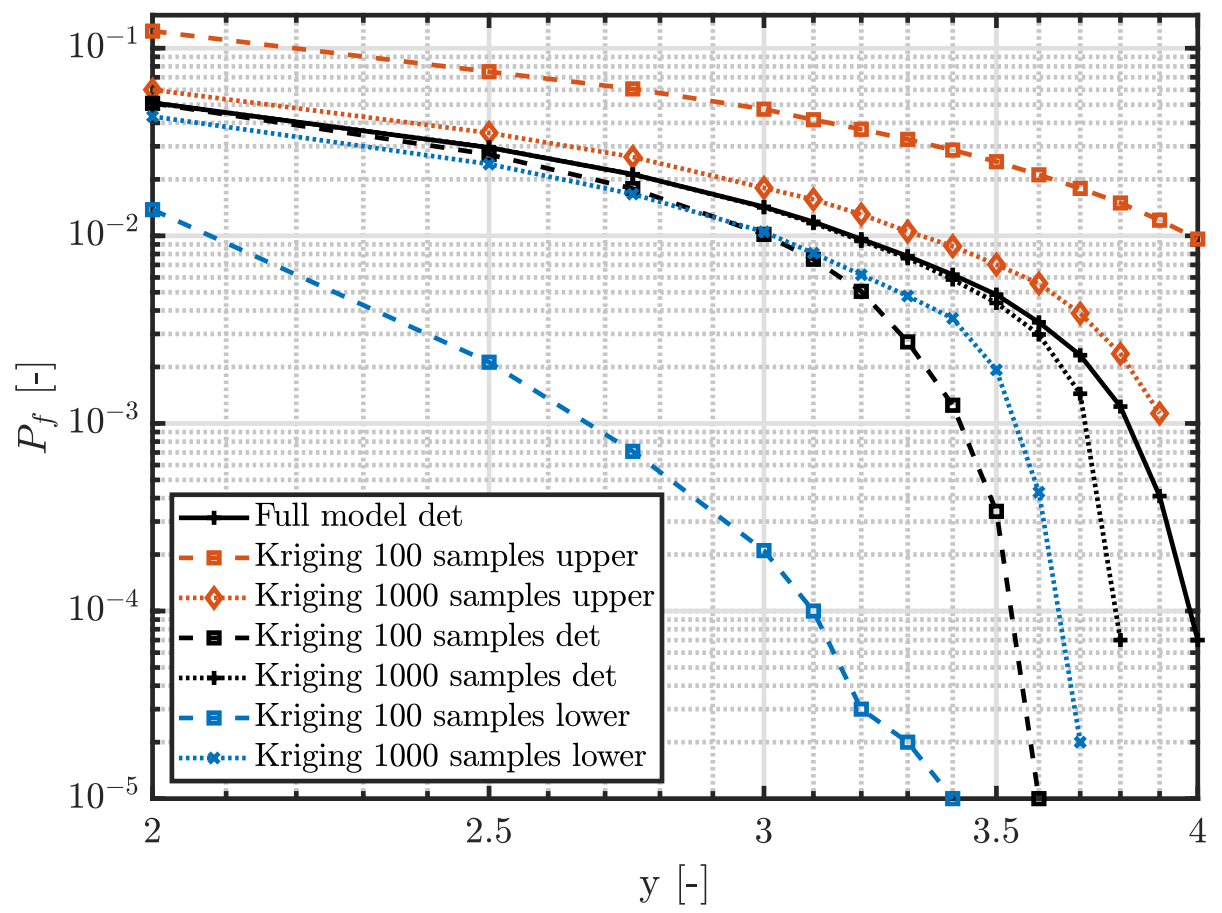

Figure 5: Performance of the Kriging surrogate models trained with different data sets in predicting the failure probability of Adjiman's function. For clarity, only the results of the models trained with 100 and 1000 are shown.

Figure 6 illustrates the performance of the interval predictor model in predicting the upper bound of $P_{f}$. Specifically, the $\pm \epsilon$ bounds on the pre- 
diction of the upper limit of the failure probability $\bar{P}_{f}$ are illustrated for all data sets. Also the prediction of the failure probability using the analytic model is illustrated. Only the upper bound of the IPM is illustrated for visualization purposes, since this is the most relevant from an engineering standpoint. First, it can be seen that except for $y=2$ and $y=2.6$, the exact failure probability always lies inside the $\epsilon$ bounds of the upper bound prediction of the IPM. Hence, the IPM always gives a safe estimation of the failure probability. However, when the true $P_{f}$ becomes smaller than 0.01 for the model trained with 1000 samples, the $\epsilon$ bounds inflate very quickly, making the estimate very conservative. This behavior is more pronounced for smaller data sets, since the confidence in the interval is proportional to the size of the training data set. Finally, it can be noted that the upper bound prediction of the set, without taking $\epsilon$ into account is more accurate than the IPM that is trained with 1000 samples. This indicates over-training of the polynomial basis, which is possibly aggravated by the iterative pruning of the polynomial basis as explained in section 2.2.

Figure 7 illustrates the performance of the adaptive Kriging model. Specifically, the $\pm 2 \cdot \sigma$ bounds are illustrated together with the crisp (mean) estimate of the model for all considered training data sets. Also the prediction of the failure probability using the analytic model is illustrated. First, the crisp estimate of the adaptive Kriging model is highly accurate for all datasets, except for the model trained with 110 samples. Furthermore, when $P_{f}<2 \cdot 10^{-04}$ the crisp accuracy degrades quickly. The $\pm 2 \cdot \sigma$ bounds on the prediction are in all cases conservative w.r.t. the actual failure probability. It can be noted that the prediction bounds of the adaptive Kriging model are 


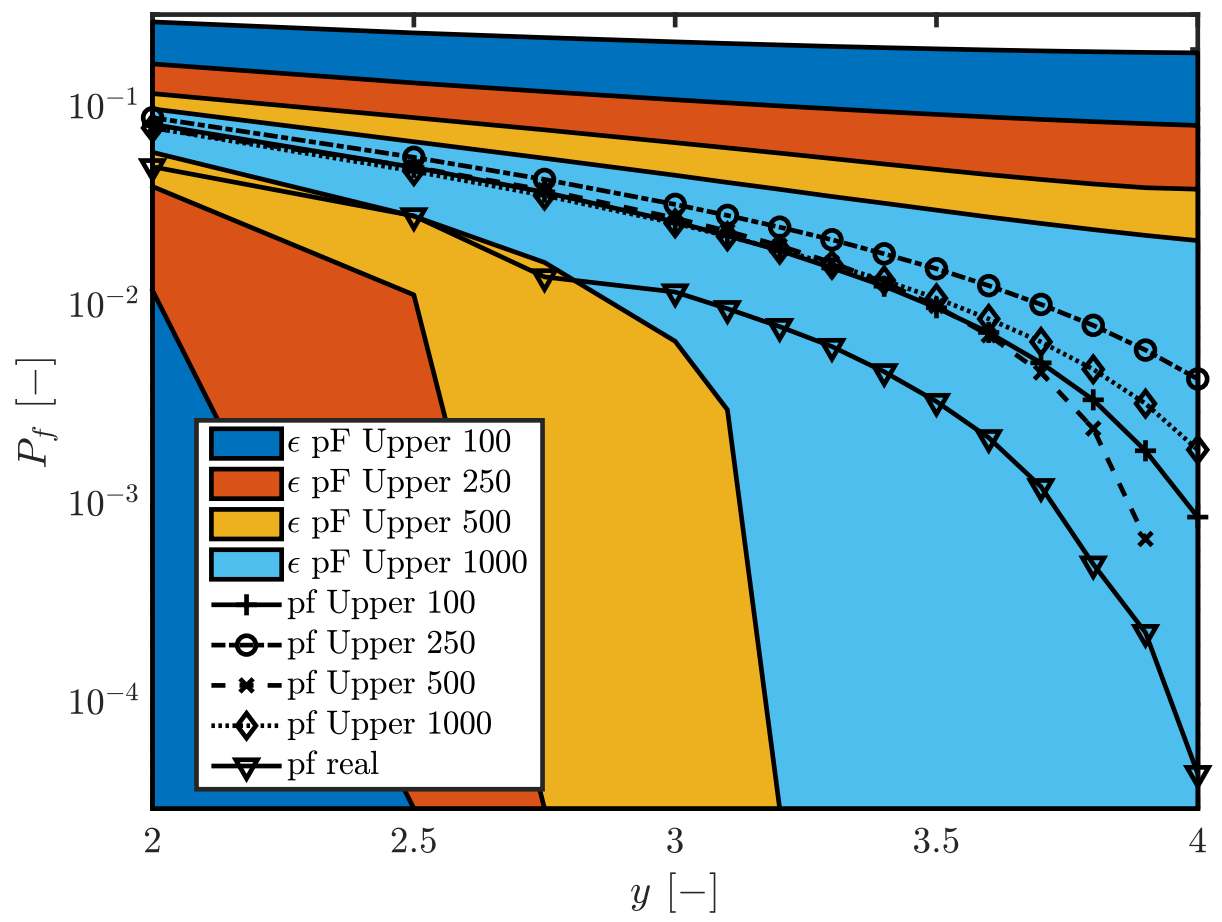

Figure 6: Performance of the IPM surrogate models trained with different data sets in predicting the failure probability of Adjiman's function.

less over-conservative as compared to the regular Kriging model. This is a direct result from the fact that the adaptive training procedure of the Kriging model directs more training points towards the zone with a high probability of failure. Therefore, given the same number of training points, the sampling will be denser in the region of the input space where the extrema of the function are locate, and as such, the variance of the Kriging estimator will locally be lower in this region.

As such, the best performing method of the considered surrogate models in terms of needed training data, accuracy and conservatism of the predicted 


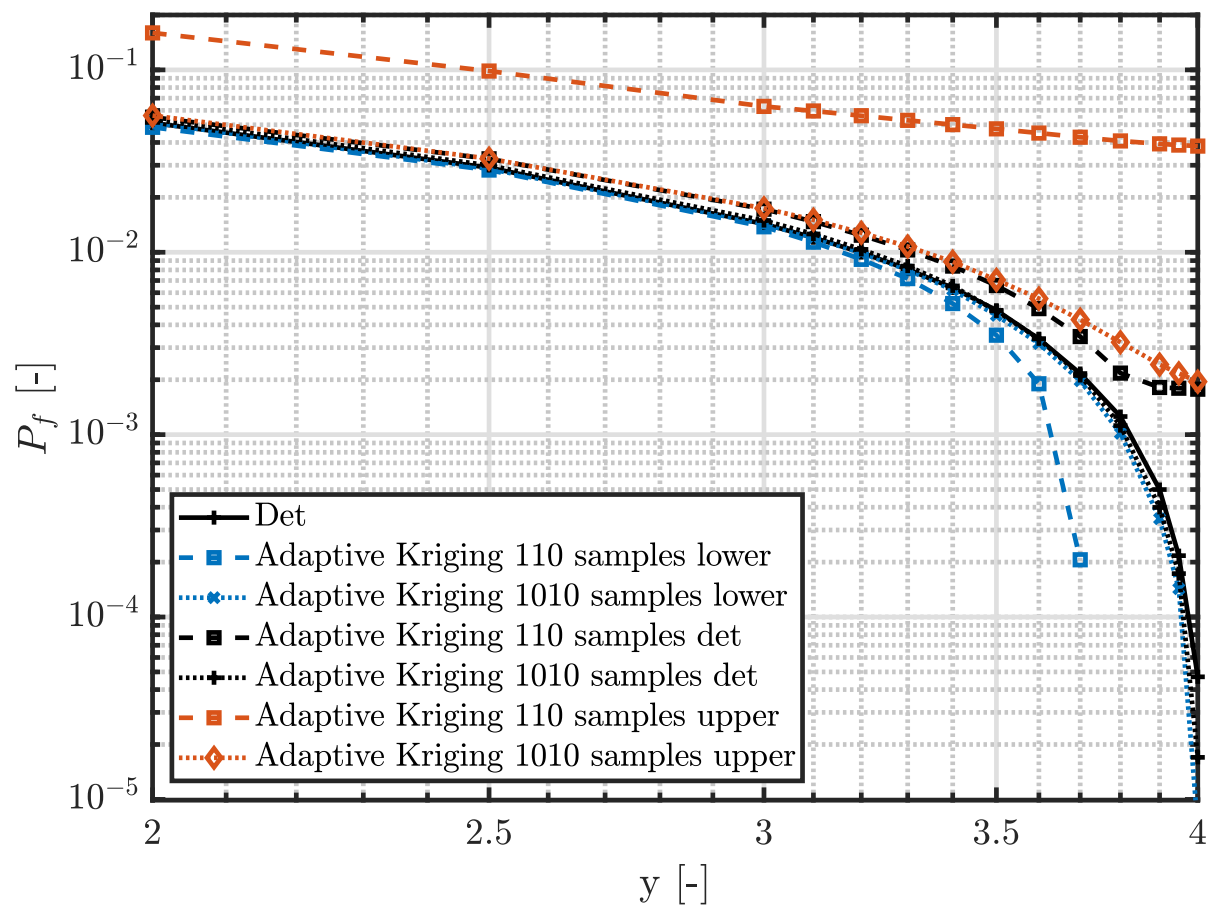

Figure 7: Performance of the Adaptive Kriging surrogate models trained with different data sets in predicting the failure probability of Adjiman's function. For clarity, only the results of the models trained with 110 and 1010 are shown

$P_{f}$ is adaptive Kriging. This can be explained by the fact that the adaptive Kriging model aims at optimizing the surrogate model performance in those regions where extrema of the model are located. However, in other regions of the model, the adaptive Kriging model generally is expected not to perform well due to a lack of local training points. In general, the improved performance of Adaptive Kriging when compared with Kriging should inspire the development of similar active learning type methods for IPMs. This is particularly important for low failure probabilities where the performance of 
the IPM is worst due to the comparatively high value of $\epsilon$.

Finally, it can be seen that by using a surrogate model, computational expenses for evaluating small failure probabilities can be decreased drasti-

cally. This statement is based on the argumentation that the application of advanced Monte Carlo methods for the estimation of small failure probabilities in conjunction with non-linear limit-state functions might prove to be computationally very demanding when a full-scale numerical model is used for the prediction of $P_{f}$.

\section{Conclusions}

In case highly non-linear limit state functions occur in the estimation of small failure probabilities, advanced Monte Carlo methods such as Line Sampling or SubSet simulation may perform poorly or may still need a large number of deterministic model evaluations to converge to a sufficiently small coefficient of variance on the estimator. Less expensive surrogate models that are calibrated in a supervised learning approach are therefore often used. However, these surrogate model prediction introduce a further level of uncertainty due to their approximative nature. This paper presents a study on the robust estimation of small failure probabilities in strong non-linear models. Specifically, Kriging and Interval Predictor Models are employed since they give an estimate of the uncertainty on the computed model response, and hence, provide the analyst with a confidence interval on the prediction. Since the intervals are used to model the uncertainty on the surrogate model estimation superpose on the propagated variability stemming from the random model parameters, the failure probability should be com- 
puted using a probability box formulation of the model response. It is shown that this problem reduces to computing two separate failure probabilities, using only a single run of model evaluations. Therefore, instead of focusing on the crisp estimate of the surrogate model to compute the probability of failure, it is suggested to take the corresponding uncertainty into account. For practical purposes, it is moreover even sufficient to consider the upper bound on the failure probability prediction. The performed case studies on a highly non-linear function show that:

- advanced Monte Carlo methods per se are sometimes not sufficient to perform accurate failure probability computations in highly non-linear models, leading to often still high computational expenses. This calls for the application of robust surrogate modeling procedures.

- Interval predictor models always provide a robust estimate of the probability of failure, yet when small failure probabilities are considered, the bounds on the prediction become non-informatively large, especially for smaller data sets. Also over-training of the polynomial basis can occur when too many data-points are provided for the training.

- The presented Adaptive Kriging method outperforms regular Kriging and Interval Predictor Models significantly in terms of over-conservatism and accuracy of the estimate and needed training data. However, it is expected that the Adaptively trained Kriging model performs poorly in other regions of the model domain due to a lack of training points. 


\section{Acknowledgements}

The Flemish Research Foundation is acknowledged for their support in the research project G0C2218N, as well as for the post-doctoral grant 12P359N of Matthias Faes.

\section{References}

\section{References}

[1] P. Koutsourelakis, H. Pradlwarter, G. Schuëller, Reliability of structures in high dimensions, part i: algorithms and applications, Probabilistic Engineering Mechanics 19 (4) (2004) 409-417.

[2] S.-K. Au, J. L. Beck, Estimation of small failure probabilities in high dimensions by subset simulation, Probabilistic engineering mechanics 16 (4) (2001) 263-277.

[3] S.-K. Au, E. Patelli, Rare event simulation in finite-infinite dimensional space, Reliability Engineering \& System Safety 148 (2016) 67-77.

[4] S. K. Au, J. L. Beck, Subset simulation and its application to seismic risk based on dynamic analysis, Journal of Engineering Mechanics 129 (8) (2003) 901-917. doi:10.1061/(ASCE)0733-9399(2003)129:8(901).

[5] G. Schuëller, H. Pradlwarter, P. Koutsourelakis, A critical appraisal of reliability estimation procedures for high dimensions, Probabilistic engineering mechanics 19 (4) (2004) 463-474. 
[6] H. Pradlwarter, M. Pellissetti, C. Schenk, G. Schuëller, A. Kreis, S. Fransen, A. Calvi, M. Klein, Realistic and efficient reliability estimation for aerospace structures, Computer Methods in Applied Mechanics and Engineering 194 (12) (2005) 1597-1617.

[7] G. Schuëller, H. Pradlwarter, Benchmark study on reliability estimation in higher dimensions of structural systems-an overview, Structural Safety 29 (3) (2007) 167-182.

[8] B. Sudret, Global sensitivity analysis using polynomial chaos expansions, Reliability Engineering \& System Safety 93 (7) (2008) 964 - 979, bayesian Networks in Dependability. doi:https://doi.org/10.1016/j.ress.2007.04.002.

URL http://www.sciencedirect.com/science/article/pii/S0951832007001329

[9] M. Moustapha, J.-M. Bourinet, B. Guillaume, B. Sudret, Comparative study of kriging and support vector regression for structural engineering applications, ASCE-ASME Journal of Risk and Uncertainty in Engineering Systems, Part A: Civil Engineering 4 (2) (2018) 04018005. arXiv:https://ascelibrary.org/doi/pdf/10.1061/AJRUA6.0000950, doi:10.1061/AJRUA6.0000950.

URL https://ascelibrary.org/doi/abs/10.1061/AJRUA6.0000950

[10] M. Broggi, M. Faes, E. Patelli, Y. Govers, D. Moens, M. Beer, Comparison of bayesian and interval uncertainty quantification: Application to the airmod test structure, in: 2017 IEEE Symposium Series on Computational Intelligence (SSCI), 2017, pp. 1-8. doi:10.1109/SSCI.2017.8280882. 
[11] M. Faes, M. Broggi, E. Patelli, Y. Govers, J. Mottershead, M. Beer, D. Moens, A multivariate interval approach for inverse uncertainty quantification with limited experimental data, Mechanical Systems and Signal Processing 118 (2019) 534-548. doi:10.1016/j.ymssp.2018.08.050.

[12] F. A. Viana, R. T. Haftka, Probability of failure uncertainty quantification with kriging, in: 53rd AIAA/ASME/ASCE/AHS/ASC Structures, Structural Dynamics and Materials Conference 20th AIAA/ASME/AHS Adaptive Structures Conference 14th AIAA, 2012, p. 1853.

[13] M. Faes, M. Broggi, M. Beer, D. Moens, Failure probability under uncertain surrogate model predictions, Proceedings of the joint ICVRAM ISUMA UNCERTAINTIES conference (2).

[14] D. G. Krige, A statistical approach to some basic mine valuation problems on the witwatersrand, Journal of the Southern African Institute of Mining and Metallurgy 52 (6) (1951) 119-139.

[15] S. N. Lophaven, H. B. Nielsen, J. Søndergaard, Dace-a matlab kriging toolbox, version 2.0, Tech. rep. (2002).

[16] R. Schöbi, B. Sudret, S. Marelli, Rare event estimation using polynomial-chaos kriging, ASCE-ASME Journal of Risk and Uncertainty in Engineering Systems, Part A: Civil Engineering 3 (2) (2016) D4016002.

[17] M. De Munck, D. Moens, W. Desmet, D. Vandepitte, An efficient response surface based optimisation method for non-deterministic har- 
monic and transient dynamic analysis, Computer Modeling in Engineering and Sciences (CMES) 47 (2) (2009) 119.

[18] M. C. Campi, G. Calafiore, S. Garatti, Interval predictor models: Identification and reliability, Automatica 45 (2) (2009) 382-392.

[19] L. G. Crespo, D. P. Giesy, S. P. Kenny, Interval predictor models with a formal characterization of uncertainty and reliability, in: Decision and Control (CDC), 2014 IEEE 53rd Annual Conference on, IEEE, 2014, pp. 5991-5996.

[20] L. G. Crespo, S. P. Kenny, D. P. Giesy, Interval predictor models with a linear parameter dependency, Journal of Verification, Validation and Uncertainty Quantification 1 (2) (2016) 021007.

[21] L. G. Crespo, S. P. Kenny, D. P. Giesy, Staircase predictor models for reliability and risk analysis, Structural Safety 75 (2018) 35-44.

[22] W. Verhaeghe, W. Desmet, D. Vandepitte, D. Moens, Interval fields to represent uncertainty on the output side of a static FE analysis, Computer Methods in Applied Mechanics and Engineering 260 (0) (2013) 50-62. doi:10.1016/j.cma.2013.03.021.

[23] M. Faes, J. Cerneels, D. Vandepitte, D. Moens, Identification and quantification of multivariate interval uncertainty in finite element models, Computer Methods in Applied Mechanics and Engineering 315 (2017) 896-920. doi:10.1016/j.cma.2016.11.023.

[24] M. Faes, D. Moens, Identification and quantification of spatial interval 
uncertainty in numerical models, Computers and Structures 192 (2017) 16-33. doi:10.1016/j.compstruc.2017.07.006.

[25] X. Liu, L. Yin, L. Hu, Z. Zhang, An efficient reliability analysis approach for structure based on probability and probability box models, Structural and Multidisciplinary Optimization 56 (1) (2017) 167-181.

[26] E. Patelli, M. Broggi, S. Tolo, J. Sadeghi, Cossan software: A multidisciplinary and collaborative software for uncertainty quantification, in: Proceedings of the 2nd ECCOMAS thematic conference on uncertainty quantification in computational sciences and engineering, UNCECOMP, 2017.

[27] H. Zhang, R. L. Mullen, R. L. Muhanna, Interval monte carlo methods for structural reliability, Structural Safety 32 (3) (2010) 183-190.

[28] C. Jiang, W. Li, X. Han, L. Liu, P. Le, Structural reliability analysis based on random distributions with interval parameters, Computers \& Structures 89 (23) (2011) 2292-2302.

[29] E. Patelli, H. M. Panayirci, M. Broggi, B. Goller, P. Beaurepaire, H. J. Pradlwarter, G. I. Schuëller, General purpose software for efficient uncertainty management of large finite element models, Finite elements in analysis and design 51 (2012) 31-48.

[30] M. de Angelis, E. Patelli, M. Beer, Advanced line sampling for efficient robust reliability analysis, Structural safety 52 (2015) 170-182. 
[31] M. De Angelis, E. Patelli, M. Beer, An efficient strategy for computing interval expectations of risk, Safety, Reliability, Risk and Life-Cycle Performance of Structures \& Infrastructures (March 2014) (2013) 978-1. 